\title{
Linggan Wuwei Jiangxin formula ameliorates airway hyperresponsiveness through suppression of IL-1 $\beta$ and IL-17A expression in allergic asthmatic mice especially with diet-induced obesity
}

\author{
Zifeng Ma ${ }^{1 \#}$, Cui Li ${ }^{1 \#}$, Lingna Xue ${ }^{1}$, Shaoyan Zhang ${ }^{1}$, Yongqing Yang ${ }^{2}$, Huiyong Zhang ${ }^{3}$, Zhenhui Lu ${ }^{1}$ \\ ${ }^{1}$ Institute of Respiratory Disease, Longhua Hospital Shanghai University of Traditional Chinese Medicine, Shanghai, China; ${ }^{2}$ Laboratory of \\ Molecular Biology, Yueyang Hospital Shanghai University of Traditional Chinese Medicine, Shanghai, China; ${ }^{3}$ Department of Respiratory, Longhua \\ Hospital Shanghai University of Traditional Chinese Medicine, Shanghai, China \\ Contributions: (I) Conception and design: H Zhang, Z Lu, Z Ma; (II) Administrative support: Y Yang, H Zhang, Z Lu; (III) Provision of study \\ materials or patients: L Xue, S Zhang, C Li; (IV) Collection and assembly of data: Z Ma, C Li; (V) Data analysis and interpretation: Z Ma; (VI) \\ Manuscript writing: All authors; (VII) Final approval of manuscript: All authors. \\ \#These authors contributed equally to this work. \\ Correspondence to: Huiyong Zhang. No. 725 South Wanping Road, No. 3 Building, Xuhui District, Shanghai, China. Email: tcmdoctorzhang@163.com; \\ Zhenhui Lu. No. 725 South Wanping Road, No. 7 Building, Xuhui District, Shanghai, China. Email: Dr_luzh@shutcm.edu.cn.
}

Background: Obese asthma represents a disease phenotype, which is associated with worse disease control and unresponsiveness to standard anti-inflammatory regimens, including inhaled corticosteroids. Obesityrelated innate airway hyperresponsiveness (AHR) plays a role in this asthma phenotype via activation of the IL-1ß/innate lymphoid cell 3 (ILC3)/IL-17A pathway. Linggan Wuwei Jiangxin (LGWWJX) formula may be a promising therapeutic option for obese asthma according to traditional Chinese medicine theory, clinical experience and related research.

Methods: The murine model of allergic asthma with obesity was induced by ovalbumin (OVA) sensitization and challenge in combination with a high fat diet (HFD). LGWWJX formula intervention was oral administrated. AHR and bronchoalveolar lavage fluid (BALF) cellularity were measured. Lung and liver histopathology assessment was performed by haematoxylin and eosin (H\&E) staining. IL-1 $\beta$ and IL-17A in BALF and serum were evaluated by ELISA. Additionally, the influence of different concentrations of LGWWJX formula on IL-1 $\beta$ stimulated IL-17A mRNA expression in ILC3 cells was evaluated in vitro.

Results: LGWWJX treatment significantly reduced AHR and allergic airway inflammatory responses in asthmatic mice, as measured by pulmonary histopathology and BALF cellularity, and these effects were more pronounced in obese asthmatic mice. While eosinophil infiltration in BALF was suppressed with LGWWJX treatment in non-obese asthmatic mice, neutrophils and basophils were significantly decreased in obese asthmatic mice. Notably, LGWWJX also demonstrated remarkable efficacy for weight loss and improvements in hepatic steatosis in mice fed with a HFD. Furthermore, the protein levels of IL-1 $\beta$ in both serum and BALF, as well as those of BALF IL-17A, declined with LGWWJX intervention in both obese and non-obese asthmatic mice, and results from ex-vivo experiments found that LGWWJX significantly attenuated the expression of IL-17A in ILC3 cells with or without stimulation by IL-1 $\beta$.

Conclusions: LGWWJX may exert a protective effect on asthmatic individuals, especially those with concurrent obesity, most likely through mechanisms including the inhibition of the IL-1ß/ILC3/IL-17A/ AHR axis, anti-inflammatory effects, weight loss, and the regulation of lipid metabolism. This suggests a promising role of LGWWJX, alone or in combination with anti-inflammatory agents, for the treatment of obese asthma.

Keywords: Linggan Wuwei Jiangxin formula (LGWWJX formula); airway hyperresponsiveness (AHR); obese asthma; IL-1 $\beta$; IL-17A 
Submitted Feb 03, 2021. Accepted for publication Apr 17, 2021.

doi: 10.21037/atm-21-1189

View this article at: http://dx.doi.org/10.21037/atm-21-1189

\section{Introduction}

The pathogenic link between obesity and asthma, as well as the concept of obesity-related asthma, has recently been fully recognized, and is characterized by pronounced respiratory symptoms and relative unresponsiveness to standardized treatment regimens (1). Weight loss through lifestyle modifications (e.g., dieting and exercise) or surgery (e.g., laparoscopic adjustable gastric banding) significantly improves lung function, asthma control, and quality of life $(2,3)$. Although multiple factors underlying the mechanisms of obese asthma have been widely explored and proposed over recent decades, which involve mechanical factors (e.g., reduction in functional residual capacity), inflammation (e.g., altered airway inflammation and chronic low-grade systemic inflammation), and the immune-metabolic axis (e.g., microbial dysbiosis, mitochondrial dysfunction, free fatty acid metabolism, and glucagon-like peptide pathway), numerous questions remain to be answered (1).

Inhaled corticosteroids (ICS), in view of their strong anti-inflammatory effects, have long been regarded as the mainstay of asthma maintenance treatment, with no other anti-inflammatory drugs superior enough to challenge its therapeutic position. However, compared with lean asthmatics, a higher proportion of patients with obesity demonstrate unresponsiveness even to regimens containing high doses of ICS or oral corticosteroids and fail to achieve good asthma control (1), leading to a pressing demand for the emergence of promising new therapeutic options for this subgroup of patients.

Both asthma and obesity have been well documented in traditional Chinese medicine since more than 2,000 years ago, along with the development of multiple therapeutic options including traditional medicines and acupuncture. The Linggan Wuwei Jiangxin (LGWWJX) formula originates from the "Synopsis of Golden Chamber" written by Zhongjing Zhang, one of the most famous Chinese doctors in the Han Dynasty. It has been used to treat cold asthma for more than 1,000 years, in part through the therapeutic principle of warming the lung to resolve fluid, along with its anti-inflammatory and antioxidative properties $(4,5)$. In addition, the typical formula of "warming yang to resolve fluid" treatment method, Linggui Zhugan (LGZG), from which LGWWJX can be processed, has been shown to attenuate high fat diet (HFD)-induced weight gain, dyslipidemia, and fatty liver disease, most likely through improving beta-oxidation, metabolism, and the transport of fatty acid $(6,7)$. Therefore, it is reasonable to speculate that LGWWJX might exert beneficial effects on asthma control, especially in overweight or obese patients.

In this study, we determined for the first time the potential protective effects of Linggan Wuwei Jiangxin formula on asthmatic mice, focusing on those with dietinduced obesity, with underlying mechinisms explored.

We present the following article in accordance with the ARRIVE reporting checklist (available at http://dx.doi. org/10.21037/atm-21-1189).

\section{Methods}

\section{Reagents}

The extracts of LGWWJX formula and its single traditional Chinese herbs without adjuvants were provided by Sichuan Neo-Green Pharmaceutical Technology Development Co., Ltd. (Chengdu, Sichuan, China). The LGWWJX formula is listed in Table 1. Standards for LGWWJX, including liquiritin, schizandrin, and deoxyschizandrin were purchased from the National Institutes for Food and Drug Control (Beijing, China), and isoliquiritin and glycyrrhizinic acid were from Sichuan Weikeqi Biological Technology Co., Ltd. (Chengdu, Sichuan, China). Rodent diets with 60 and $10 \mathrm{kcal} \%$ fat were purchased from Research Diets, Inc. (New Brunswick, NJ, USA) and Jiangsu Synergetic Pharmaceutical Bioengineering Co., Ltd. (Nanjing, Jiangsu, China), respectively. Ovalbumin (OVA) was purchased from Sigma-Aldrich (St. Louis, MO, USA). Enzyme-linked immunosorbent assay (ELISA) kits for IL-1 $\beta$ and IL-17A were purchased from R\&D Systems (Minneapolis, MN, USA). Recombinant mouse IL-1 $\beta$ and IL-1 $\beta$ receptor antagonists were purchased from Kingfisher Biotech, Inc. (St. Paul, MN, USA). Collagenase IV and DNase I were purchased from Sigma-Aldrich (St. Louis, MO, USA). Percoll was purchased from GE Healthcare (Pittsburgh, PA, USA). Purified anti-mouse CD16/32 and Lineage (Lin) cocktail markers were purchased from Biolegend (San Diego, CA, USA). The Foxp3 staining kit, Live/Dead Fixable viability dyes (FVD), and ROR $\gamma \mathrm{T}$, CD 90.2 (Thy-1.2), CD 45 monoclonal antibodies were 
Table 1 The composition of the Linggan Wuwei Jiangxin (LGWWJX) formula

\begin{tabular}{llll}
\hline $\begin{array}{l}\text { Chinese } \\
\text { name }\end{array}$ & Latin name & English name & Amount (g) \\
\hline Fu Ling & Poria & Indian Buead & 12 \\
Gan Cao & Radix Glycyrrhizae & Liquorice Root & 6 \\
Gan Jiang & Rhizoma Zingiberis & Dried Ginger & 9 \\
Xi Xin & Herba Asari & Manchurian & 3 \\
& & Wildginger & \\
Wu Wei Zi & Fructus Schisandrae & $\begin{array}{l}\text { Chinese } \\
\text { Magnoliavine Fruit }\end{array}$ \\
\hline
\end{tabular}

purchased from eBioscience (San Diego, CA, USA). The lactate dehydrogenase (LDH) Cytotoxicity Assay Kit was purchased from Beyotime (Shanghai, China). Total RNA isolation reagent was purchased from Tiangen Biotech Co., Ltd. (Beijing, China). The Prime Script RT Master Mix kit (Perfect Real Time) and Universal SYBR Master Mix were purchased from TaKaRa (Tokyo, Japan). The primers for IL17A (Fwd: 5'-ACTACCTCAACCGTTCCACG-3'; Rev: 5'-TTCCCTCCGCATTGACACAG-3') and GAPDH (Fwd: 5'-AGGTCGGTGTGAACGGATTTG-3'; Rev: 5 '-TGTAGACCATGTAGTTGAGGTCA-3') were synthesized by Shanghai Yanjiang Biotechnology Co., Ltd. (Shanghai, China).

\section{High performance liquid chromatography (HPLC) analysis of LGWW'X formula}

HPLC analysis was performed using a Phenomenex Gemini chromatographic column at the detection wavelength of $260 \mathrm{~nm}$, with a mixture of formic acid water (A)-acetonitrile (B) as the mobile phase. A linear gradient elution was conducted as follows: $0-45 \min : 10 \% \mathrm{~B} \rightarrow 25 \% \mathrm{~B}, 45-65 \mathrm{~min}: 25 \% \mathrm{~B} \rightarrow$ $32 \%$ B, $65-80$ min: $32 \%$ B $\rightarrow 35 \%$ B, $80-120 \mathrm{~min}: 35 \%$ B $\rightarrow$ $70 \% \mathrm{~B}, 120-130 \mathrm{~min}: 70 \% \mathrm{~B}$. The flow rate was $1 \mathrm{~mL} / \mathrm{min}$ and the injection volume was $10 \mu \mathrm{L}$. Column temperature was constantly kept at $30^{\circ} \mathrm{C}$. The HPLC analysis of standards (liquiritin, isoliquiritin, glycyrrhizinic acid, schizandrin, and deoxyschizandrin) and the samples were carried out under the same experimental conditions as mentioned above.

\section{Animal experiments}

Male specific pathogen-free BALB/c mice aged
3-4 weeks were purchased from Shanghai Slake Laboratory Animal Co., Ltd. (Shanghai, China) and maintained in the Experimental Animal Center of Fudan University under controlled conditions with a temperature of $24-26^{\circ} \mathrm{C}$ and humidity of $60 \% \pm 5 \%$, and a 12 -hour light/dark cycle. A total of 4-8 mice were kept in a pathogen-free cage and were provided with ad libitum access to rodent commercial chow and sterile water. The body weights of mice were measured and recorded every week. All the procedures of experiments were approved by the Animal Welfare and Ethics Group of Department of Laboratory Animal Science Fudan University (approval number: 202003003Z), in compliance with national science and technology commission of China guidelines for the care and use of animals.

\section{Experimental design}

As shown in Figure S1, 64 mice were equally divided into 8 groups, namely the non-obese control (NC) group, non-obese control treatment (NCT) group, non-obese asthma (NA) group, non-obese asthma treatment (NAT) group, obese control (OC) group, obese control treatment (OCT) group, obese asthma (OA) group, and obese asthma treatment (OAT) group. Mice in the 4 non-obese groups were fed on a standard diet, and those in all obese groups received a HFD, starting from day 0 . To establish the mouse model of chronic asthma, mice were sensitized by intraperitoneal injection of $20 \mu \mathrm{g}$ OVA plus $2 \mathrm{mg}$ aluminium hydroxide dissolved in $200 \mu \mathrm{L}$ phosphate-buffered saline (PBS) at days 49, 56, 63, and 70, and 3 days after the last sensitization injection, mice were challenged by exposure to $3 \%$ aerosolized OVA in PBS for half an hour every second day for 4 weeks. In the 4 non-asthmatic groups, only PBS was delivered. Administration of LGWWJX $(2 \mathrm{~g} / \mathrm{Kg})$ or PBS by gavage was started at day 72 (the day before the first challenge) once daily for 4 consecutive weeks in the treatment and non-treatment groups, respectively.

\section{Pulmonary function tests}

At day 101, mice were anesthetized with $50 \mathrm{mg}$ per $\mathrm{kg}$ body weight pentobarbital, and tracheostomized, intubated, and mechanically ventilated at a tidal volume of $0.2 \mathrm{~mL}$ and a frequency of 150 breaths per min, as previously described (8). AHR, as measured by lung resistance $\left(\mathrm{R}_{\mathrm{L}}\right)$, was measured using invasive BUXCO (BUXCO Electronics) in response to increasing doses of aerosolized methacholine (Mch). 


\section{Bronchoalveolar lavage fluid (BALF) cellularity}

After blood collection, the mouse trachea was intubated and the right lung was ligated. Lavage was performed 3 times with $0.3 \mathrm{~mL}$ aliquots of PBS through the tracheal cannula (total volume $0.9 \mathrm{~mL}$ ). The collected BALF was centrifuged at $1,000 \times \mathrm{g}$ for 10 minutes at $4{ }^{\circ} \mathrm{C}$. The pelleted cells were resuspended in $0.1 \mathrm{~mL}$ of PBS containing $1 \%$ BSA, and the BALF leukocyte count was performed with the MINDRAY BC-5300vet five-class animal blood cell counter. Supernatants were collected for further analysis of cytokine concentrations.

\section{Lung and liver histopathology}

The lung and liver tissues harvested from mice were fixed with $10 \%$ neutral formalin and then embedded in paraffin. Afterwards, sections were stained with haematoxylin and eosin (H\&E) for histological assessment.

\section{Measurement of IL-1ß and IL-17A in BALF and serum}

The concentrations of IL-1 $\beta$ and IL-17A in BALF and serum were determined by ELISA using corresponding commercial kits, according to the manufacturer's instructions.

\section{Isolation of intestinal lamina propria lymphocytes (LPLs)}

LPLs were isolated as previously described, with minor modifications (9). Briefly, small or large intestines were dissected from $\mathrm{Rag}^{-/-}$mice, kindly gifted by the ILC3 research group of Shanghai Institute of Nutrition and Health (Chinese Academy of Sciences), with fat tissues and peyer's patches removed. Intestines were then cut into $2 \mathrm{~cm}$ long pieces, washed, and shaken in PBS containing $1 \mathrm{mM}$ DTT for $10 \mathrm{~min}$ at room temperature. Intestines were incubated and shaken in PBS containing $30 \mathrm{mM}$ EDTA at $37^{\circ} \mathrm{C}$ for $10 \mathrm{~min}$ for 2 cycles. The tissues were then digested in RPMI 1640 medium containing $150 \mu \mathrm{g} / \mathrm{mL}$ DNase I and $200 \mathrm{U} / \mathrm{mL}$ collagenase VIII at $37{ }^{\circ} \mathrm{C}$ in a $5 \% \mathrm{CO}_{2}$ incubator for $1.5 \mathrm{~h}$. The digested tissues were homogenized by vigorous shaking and passed through a $100 \mu \mathrm{m}$ cell strainer. And then mononuclear cells were harvested from the interphase of an $80 \%$ and $40 \%$ Percoll gradient after spinning at 2,500 rpm for $20 \mathrm{~min}$ at room temperature.

\section{ILC3 sorting and identification}

Single-cell suspensions were first stained with FVD (APC-Cy7, diluted 1:250) to exclude dead cells. Fc receptor blocking was performed using the purified anti-mouse CD16/CD32 prior to the surface staining. Then, cells were stained with CD45 (APC, diluted 1:100), CD90.2 (FITC, diluted 1:100) and Lin markers defined as CD3, Ly-6G/Ly-6C, CD11b, CD45R/B220 and Ter-119 (BV421, diluted 1:100). Another four preparations were mono-stained with FVD, CD45, CD90.2 and CD11b respectively for compensation controls. ILC3 cells were sorted as Lin $\mathrm{CD}^{-} 5^{\text {low }} \mathrm{CD} 90.2^{\text {high }}$ cells. The Foxp3 Staining kit was used for cellular fixation and nuclear ROR $\gamma \mathrm{t}$ (PE, diluted 1:50) staining for ILC3 cells identification. Flow-cytometric analysis was undertaken using a BD Fortessa X20 flow cytometer and cell sorting was done using FACS Aria II (both BD Biosciences). The representative intranuclear staining results of ROR $\gamma t$ are shown in Figure S2. The purity of sorted ILC3 cells was more than $95 \%$.

\section{In vitro cell viability assays}

The influence of LGWWJX on ILC3 cell viability was determined with a LDH kit according to the manufacturer's instructions. ILC3 cells were added into 24 -well plates $\left(10^{5}\right.$ cells per well) and incubated in a series of 10 -fold diluted LGWWJX formula for $12 \mathrm{~h}$. The supernatants were harvested for the LDH assay.

\section{The dose-response curve of the effect of IL-1及 on IL-17A production by ILC3 cells}

Recombinant IL- $1 \beta$ at the concentration of $10 \mathrm{ng} / \mathrm{mL}$ was added to stimulate the sorted ILC 3 cells $\left(10^{5}\right.$ cells per well $)$ respectively for $0,2,4$ and $6 \mathrm{~h}$. Total RNA was isolated from the collected ILC3 cells using the total RNA isolation reagent, and $0.5 \mu \mathrm{g}$ total RNA was reverse transcribed in a final volume of $20 \mu \mathrm{L}$ using random primers under standard conditions with the Prime Script RT Master Mix kit according to the manufacturer's instructions. The cDNA was amplified in the presence of the Universal SYBR qPCR Master Mix. Gene-specific PCR products were measured using the ABI 7500 System. The levels of target gene expression were normalized to GAPDH expression.

\section{The influence of different concentrations of LGWWJX

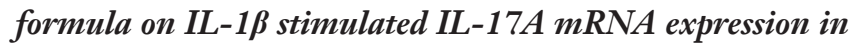 ILC3 cells}

The sorted ILC3 cells $\left(10^{5}\right.$ cells per well) were divided into 7 groups and treated, respectively, with vehicle (blank), 


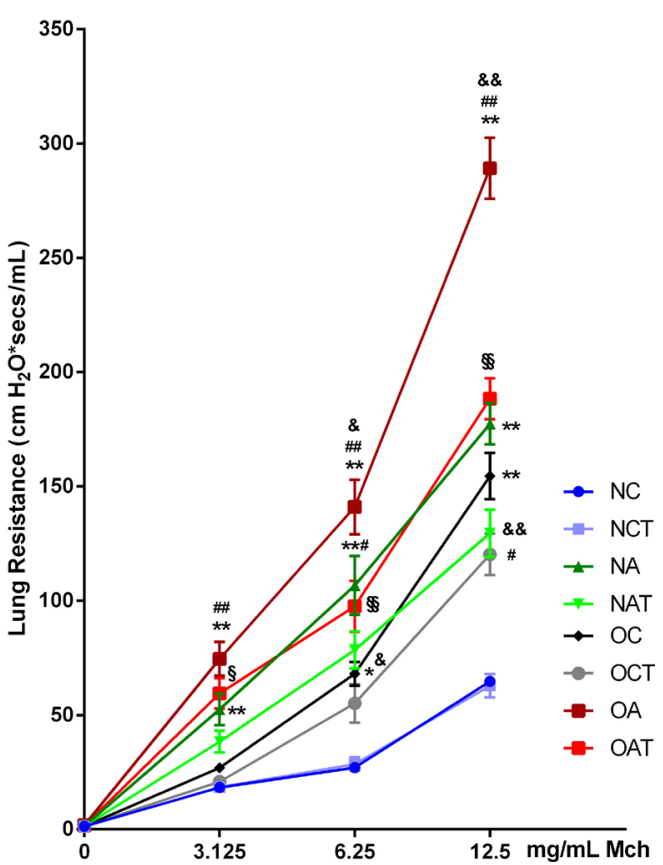

Figure 1 Effects of Linggan Wuwei Jiangxin (LGWWJX) on augmented airway hyperresponsiveness (AHR) in response to high fat diet (HFD) and/or ovalbumin (OVA) sensitization and challenge. The values are presented as mean $\pm \operatorname{SEM}(\mathrm{n}=8) .{ }^{*} \mathrm{P}<0.05$ and ${ }^{* *} \mathrm{P}<0.01$ compared with the non-obese control $(\mathrm{NC})$ group; ${ }^{*} \mathrm{P}<0.05$ and ${ }^{\# \#} \mathrm{P}<0.01$ compared with the obese control (OC) group; ${ }^{8} \mathrm{P}<0.05$ and ${ }^{\& \&} \mathrm{P}<0.01$ compared with the non-obese asthma (NA) group; ${ }^{\S} \mathrm{P}<0.05$ and ${ }^{\S \S} \mathrm{P}<0.01$ compared with the obese asthma (OA) group.

$10 \mathrm{ng} / \mathrm{mL}$ IL-1 $\beta, 250 \mu \mathrm{g} / \mathrm{mL}$ LGWWJX formula, $25 \mu \mathrm{g} / \mathrm{mL}$ LGWWJX formula, $250 \mu \mathrm{g} / \mathrm{mL}$ LGWWJX formula plus $10 \mathrm{ng} / \mathrm{mL}$ IL-1 $\beta, 25 \mu \mathrm{g} / \mathrm{mL}$ LGWWJX formula plus $10 \mathrm{ng} / \mathrm{mL} \mathrm{IL}-1 \beta$, and $10 \mathrm{ng} / \mathrm{mL} \mathrm{IL}-1 \beta$ plus IL-1R antagonist for $4 \mathrm{~h}$. Then, cell pellets were collected and washed once with PBS for assay of IL-17A mRNA expression with RT-PCR.

\section{Statistical analysis}

Statistical analysis was carried out with SPSS version 20.0 (IBM Corporation, Chicago, IL, USA). Data were expressed as mean \pm SEM and were analyzed by analysis of variance (ANOVA), followed by Tukey post hoc tests. P values of $<0.05$ were considered statistically significant.

\section{Results}

\section{HPLC profile of $L G W W 7 X$}

The HPLC fingerprint profile of the LGWWJX formula was obtained and compared with that of standard reference compounds, which confirmed the presence of liquiritin, isoliquiritin, glycyrrhizinic acid, schizandrin, and deoxyschizandrin as bioactive ingredients in the LGWWJX formula (Figure S3).

\section{LGWWFX significantly reduced AHR especially in obese asthmatic mice}

As shown in Figure 1, OVA sensitization and provocation successfully induced increased $\mathrm{R}_{\mathrm{L}}$ in both normal-weight and obese mice in the presence of increasing doses of Mch $(\mathrm{P}<0.05)$. Further elevation in $\mathrm{R}_{\mathrm{L}}$ was observed in obese versus non-obese asthmatic mice $(\mathrm{P}<0.05)$. The airway resistance of obese asthmatic mice increased more significantly than obese non-asthmatic mice $(\mathrm{P}<0.01)$. Importantly, LGWWJX markedly alleviated augmented airway responsiveness to Mch at the concentrations of 3.125, 6.25 , and $12.5 \mathrm{mg} / \mathrm{mL}$ in obese asthmatic mice $(\mathrm{P}<0.05$ or $\mathrm{P}<0.01$ ), but appeared less effective when administered to non-obese asthmatic compared with those obese mice, as the reduction in $\mathrm{R}_{\mathrm{L}}$ reached statistical significance only at the highest concentration of Mch $(\mathrm{P}<0.05)$. Notably, innate AHR was recorded in obese non-asthmatic mice in this study as expected, and significant response to LGWWJX treatment occurred only at the highest concentration of Mch $(\mathrm{P}<0.05)$.

\section{LGWWFX significantly inbibited airway inflammation in asthmatic mice}

Both obese and non-obese asthmatic mice demonstrated robust airway lumen infiltration of a variety of inflammatory cells, including neutrophils, eosinophils, basophils, and lymphocytes in BALF compared with their respective non-asthmatic mice (Figure $2 A, B, C, D, E, F)$. Eosinophils in normal-weight asthmatic mice increased significantly compared with those in obese asthmatic mice (Figure $2 E$, $\mathrm{P}<0.05)$, while the latter demonstrated more greater increases in neutrophils, lymphocytes and basophils (Figure $2 B, C, F, \mathrm{P}<0.01$ or 0.05 ). Specifically, LGWWJX significantly reduced the number of eosinophils in nonobese mice and the number of neutrophils and basophils in obese asthmatic mice (Figure $2 B, E, F, P<0.01$ or 0.05 ).

This was accompanied by representative $H \& E$ staining of mouse lung tissues as shown in Figure 3, including A-H. All asthmatic mice showed pathological manifestations of bronchial wall thickening, mucous hypersecretion, lumen 

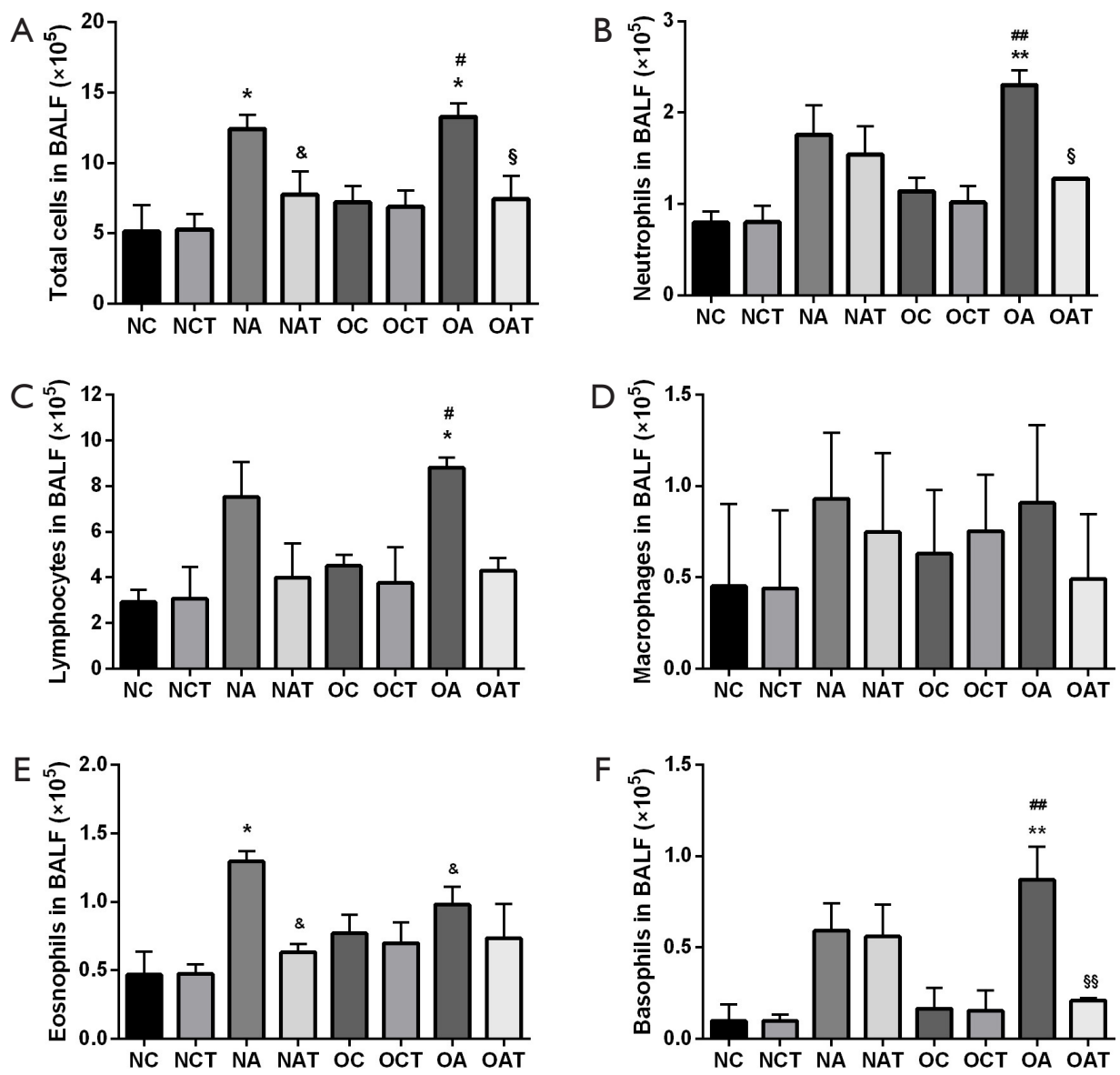

Figure 2 Effects of Linggan Wuwei Jiangxin (LGWWJX) on bronchoalveolar lavage fluid (BALF) total cells (A), and neutrophil (B), lymphocyte (C), macrophage (D), eosinophil (E), and basophil (F) differential cell counts in response to high fat diet (HFD) and/or ovalbumin (OVA) sensitization and challenge. The values are presented as mean $\pm \mathrm{SEM}(\mathrm{n}=8)$. ${ }^{*} \mathrm{P}<0.05$ and ${ }^{* *} \mathrm{P}<0.01$ compared with the non-obese control (NC) group; ${ }^{\#} \mathrm{P}<0.05$ and ${ }^{\# \#} \mathrm{P}<0.01$ compared with the obese control (OC) group; ${ }^{8} \mathrm{P}<0.05$ compared with the non-obese asthma (NA) group; ${ }^{\S} \mathrm{P}<0.05$ and ${ }^{\S} \mathrm{P}<0.01$ compared with the obese asthma (OA) group.

narrowing, and peribronchial infiltration with inflammatory cells, especially in obese asthmatic mice, as shown in (Figure $3 C, G)$. Obese mice showed obvious inflammatory cell infiltration (Figure 3E). Furthermore, LGWWJX alleviated the pathological manifestations of lung tissues from both non-obese and obese mice sensitized and challenged with OVA (Figure 3D,F,H).

\section{LGWWJX significantly decreased serum and BALF IL- $1 \beta$ and IL-17A protein levels in asthmatic mice and obese non-astbmatic mice}

The IL-1ß/ILC3/IL-17A pathway activation in vivo was explored by determining the concentrations of IL- $1 \beta$ and IL-17A in serum and BALF. As shown in Figure 4, IL-1 $\beta$ and IL-17A protein levels in both serum and BALF were enhanced through HFD and OVA challenge alone $(\mathrm{P}<0.01)$. Further increases were documented in obese $v s$. non-obese asthmatic mice $(\mathrm{P}<0.01)$, except serum IL-17A $(\mathrm{P}>0.05)$. IL-1 $\beta$ and IL-17A protein levels in both serum and BALF were reduced with LGWWJX intervention in both obese and non-obese asthmatic mice $(\mathrm{P}<0.05)$, as well as in nonasthmatic obese mice $(\mathrm{P}<0.05$ or $\mathrm{P}<0.01)$, except for serum IL-17A $(\mathrm{P}>0.05)$.

\section{LGWW7X moderately inbibited weight gain and significantly improved fat deposition in the liver tissue of HFD fed obese mice}

Mice fed with a HFD, irrespective of OVA challenge or not, 


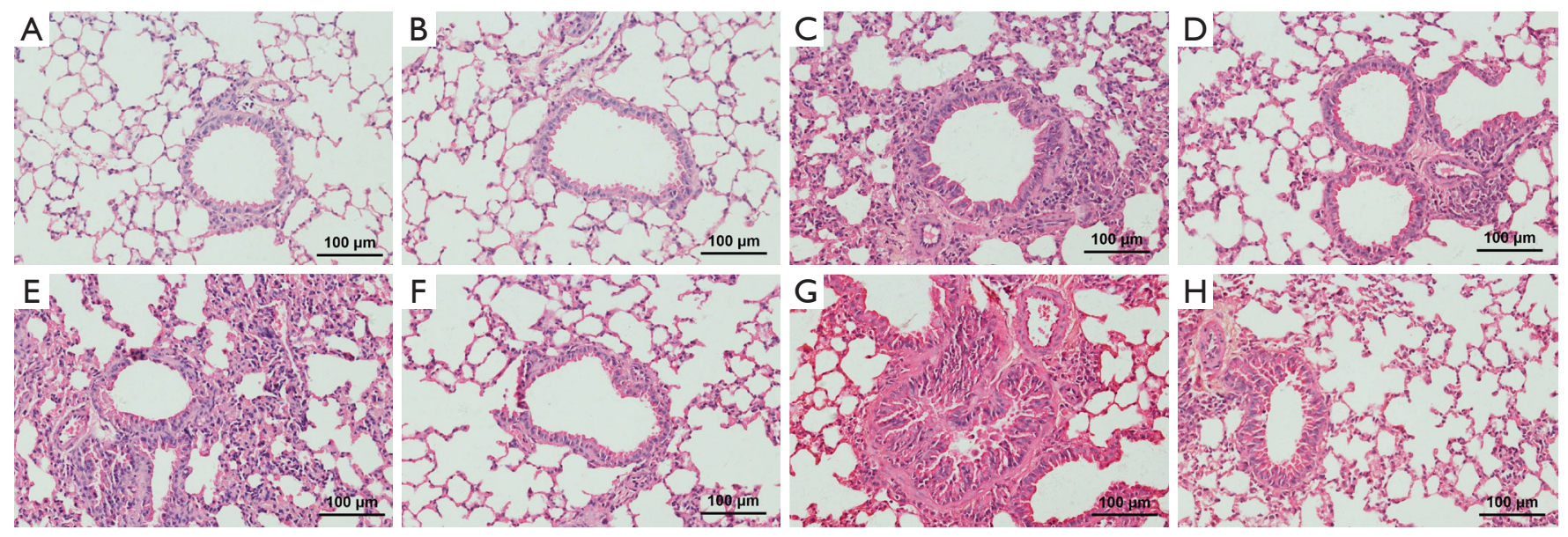

Figure 3 Representative images of haematoxylin and eosin (H\&E) staining of mouse lung tissue sections, from the (A) non-obese control (NC) group, (B) non-obese treatment (NCT) group, (C) non-obese asthma (NA) group, (D) non-obese asthma treatment (NAT) group, (E) obese control (OC) group, (F) obese control treatment (OCT) group, (G) obese asthma (OA) group, and (H) obese asthma treatment (OAT) group. Black scale bars represent $100 \mu \mathrm{m}$.
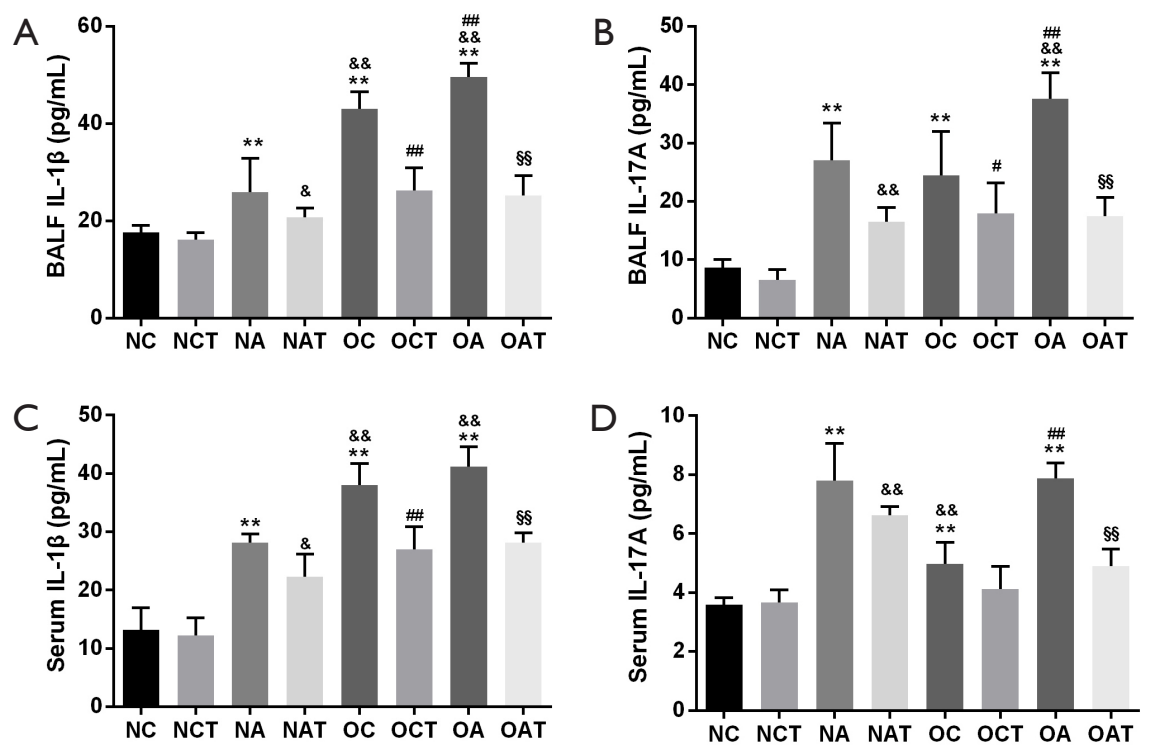

Figure 4 Effects of Linggan Wuwei Jiangxin (LGWWJX) on IL-1 $\beta$ protein levels in BALF (A) and serum (C), and IL-17A in bronchoalveolar lavage fluid (BALF) (B) and serum (D), in response to high fat diet (HFD) and/or ovalbumin (OVA) sensitization and challenge. The values are presented as mean $\pm \mathrm{SEM}$ ( $\mathrm{n}=8$ per group). ${ }^{* *} \mathrm{P}<0.01$ compared with the non-obese control (NC) group; ${ }^{*} \mathrm{P}<0.05$ and ${ }^{\# \#} \mathrm{P}<0.01$ compared with the obese control (OC) group; ${ }^{8} \mathrm{P}<0.05$ and ${ }^{\& \& \&} \mathrm{P}<0.05$ compared with the non-obese asthma (NA) group; ${ }^{\text {ss }} \mathrm{P}<0.01$ compared with the obese asthma (OA) group.

began to significantly gain weight compared to those on a normal diet at week 4 (Figure $5 A, \mathrm{P}<0.05$ ), accompanied by overt hepatic steatosis as seen with $\mathrm{H} \& \mathrm{E}$ staining (Figure 5B, a-h). Moreover, LGWWJX reduced the rate of weight gain in mice fed with a HFD with the increase in age
(Figure $5 A, \mathrm{P}<0.05)$. The numbers and sizes of cytoplasmic vacuoles in hepatocytes from these mice also reduced with this intervention (Figure $5 B, \mathrm{f} \& \mathrm{~h}$ ). In contrast, similar trends were not observed in mice on a normal diet (Figure $5 A, \mathrm{P}>0.05$; Figure 5B, b \& d). 
A

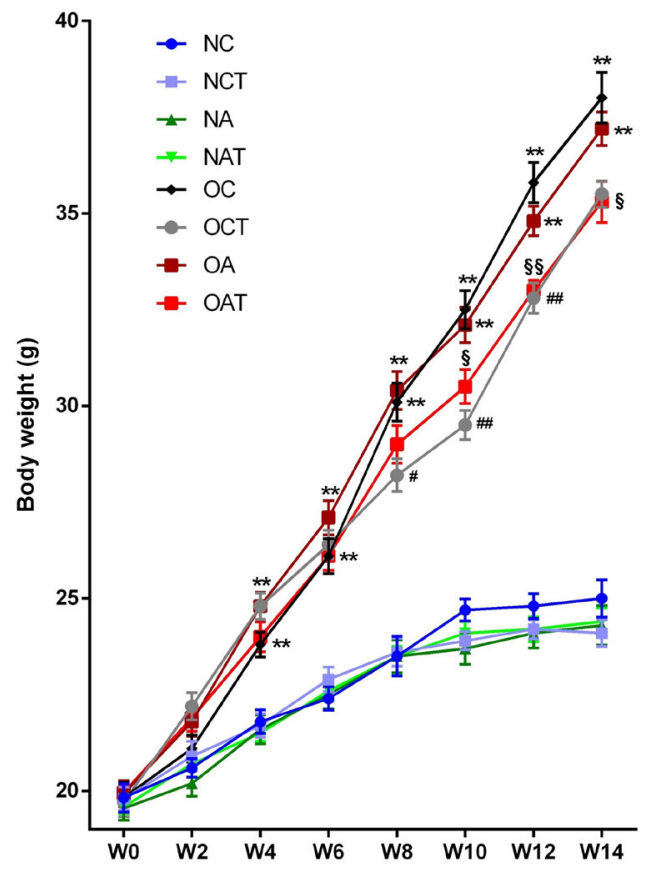

$\mathrm{B}$ a

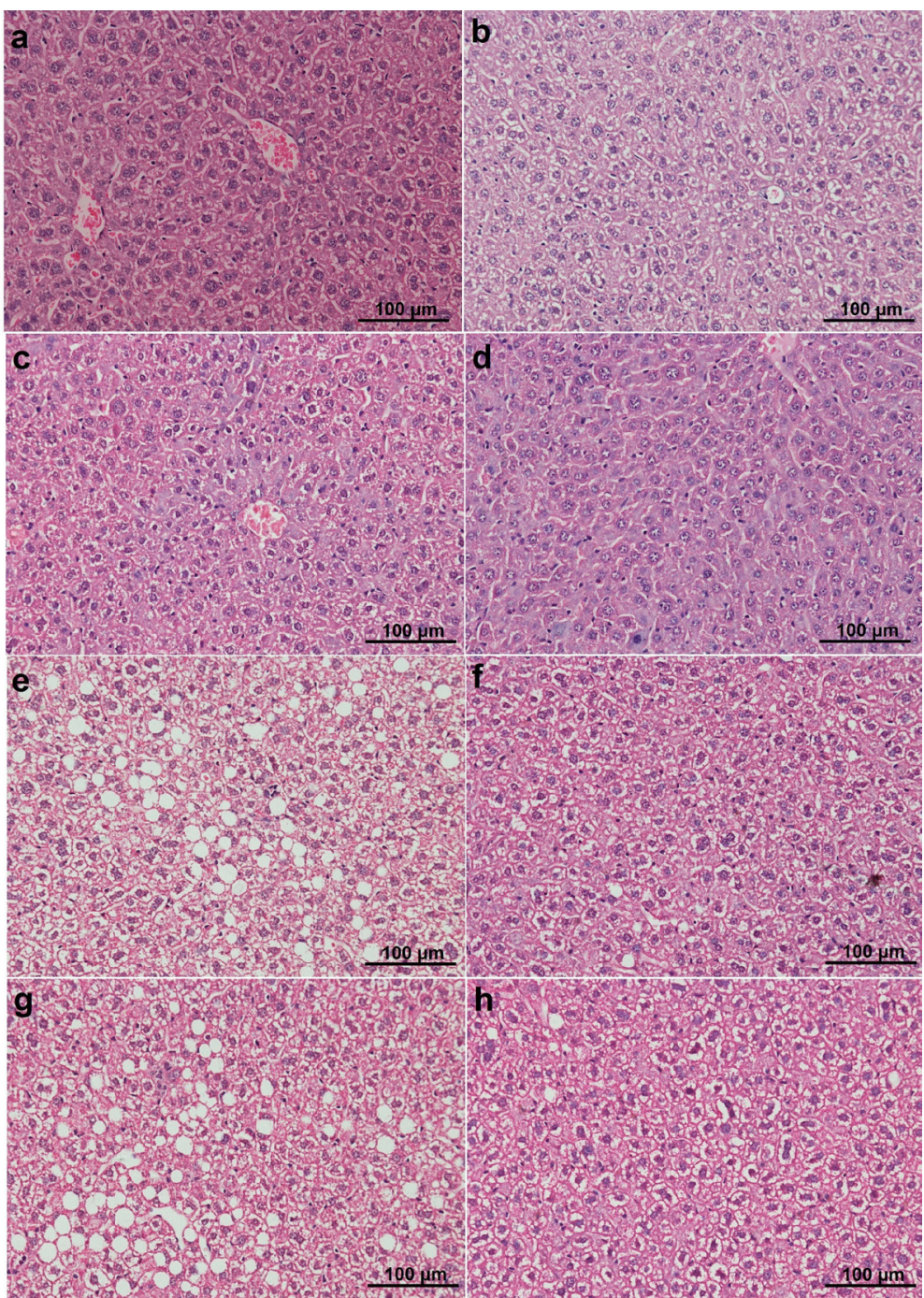

Figure 5 Effects of Linggan Wuwei Jiangxin (LGWWJX) on body weight and liver pathology in response to high fat diet (HFD) in mouse model. (A) Body weight gain in each group of mice. The values are presented as mean $\pm \operatorname{SEM}(\mathrm{n}=8)$. ${ }^{* *} \mathrm{P}<0.01$ compared with the non-obese control (NC) group; ${ }^{\#} \mathrm{P}<0.05$ and ${ }^{\# \#} \mathrm{P}<0.01$ compared with the obese control (OC) group; ${ }^{\S} \mathrm{P}<0.05$ and ${ }^{\varsigma \varsigma} \mathrm{P}<0.01$ compared with the obese asthma (OA) group. (B) Representative images of haematoxylin and eosin (H\&E) staining of mouse hepatic tissue sections, from the (a) non-obese control (NC) group, (b) non-obese treatment (NCT) group, (c) non-obese asthma (NA) group, (d) non-obese asthma treatment (NAT) group, (e) obese control (OC) group, (f) obese control treatment (OCT) group, (g) obese asthma (OA) group, and (h) obese asthma treatment (OAT) group. Black scale bars represent $100 \mu \mathrm{m}$.

\section{The in vitro cytotoxicity test of LGWWYX in ILC3 cells}

Cell viability was $100 \%$ with concentrations of LGWWJX ranging from 0.25 to $250 \mu \mathrm{g} / \mathrm{mL}$, which dropped to approximately $60 \%$ and $10 \%$ at the concentrations of 2,500 and 25,000 $\mathrm{\mu g} / \mathrm{mL}$, respectively (Figure S4). Accordingly, concentrations of $250 \mu \mathrm{g} / \mathrm{mL}$ or less were used in the subsequent in vitro experiments.

\section{The time-dependent effect of $I L-1 \beta$ on the expression of IL-17A in ILC3 cells}

IL-17A mRNA expression in ILC3 cells was examined via RT-PCR at 0,2 , 4, and $6 \mathrm{~h}$ after administration of recombinant IL-1 $\beta$. As shown in Figure 6A, the expression of IL-17A mRNA increased markedly at $4 \mathrm{~h}(\mathrm{P}<0.05)$, and declined at the $6 \mathrm{~h}(\mathrm{P}>0.05)$. Accordingly, ILC3 cells were 

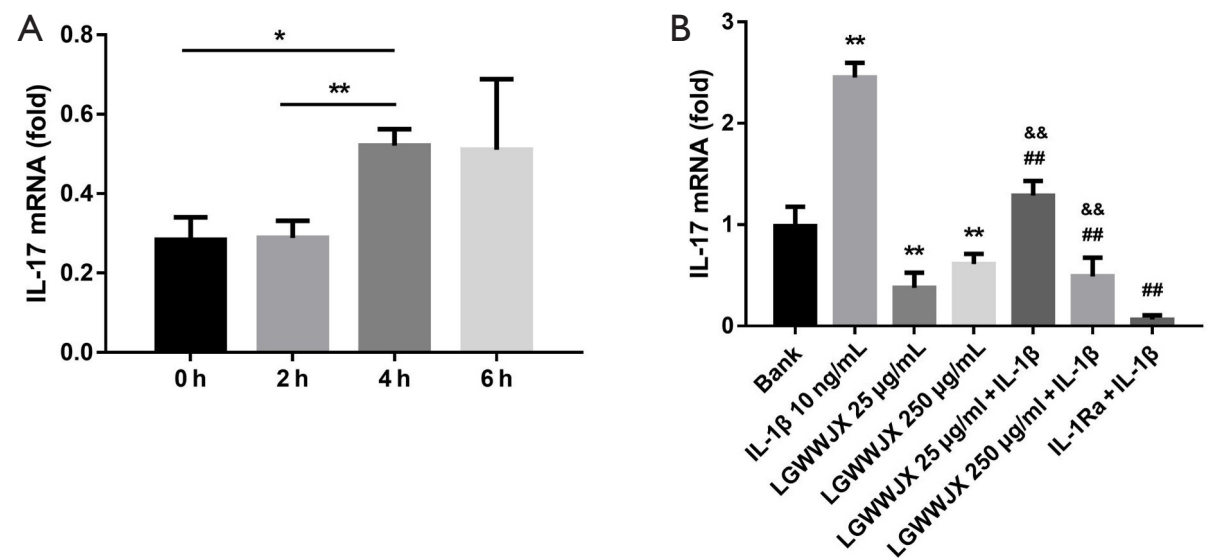

Figure 6 The effect of Linggan Wuwei Jiangxin (LGWWJX) on IL-17A production in innate lymphoid cells (ILC3 cells) induced by IL$1 \beta$ in vitro. (A) The time-dependent effects of IL-1 $\beta$ on IL-17A mRNA expression in ILC3 cells. The values are presented as mean \pm SEM. ${ }^{*} \mathrm{P}<0.05$ and ${ }^{* *} \mathrm{P}<0.01$ compared with the IL-17A mRNA level at $4 \mathrm{~h}$ post administration. (B) The effect of LGWWJX on IL-1 $\beta$-induced IL-17A production in ILC3 cells. The values are presented as mean $\pm \mathrm{SEM}$. ${ }^{* *} \mathrm{P}<0.01$ compared with the blank group; ${ }^{\# \#} \mathrm{P}<0.01$ compared with the IL- $1 \beta$ group; ${ }^{\& \&} \mathrm{P}<0.01$ compared with the IL-1Ra+IL-1 $\beta$ group.

incubated with LGWWJX for 4 hours in the next in vitro experiments.

\section{The effect of LGWWFX on IL-17A production in ILC3

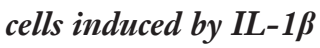

As shown in Figure $6 B$, after stimulation by IL-1 $\beta$, the IL17A expression of ILC3 was significantly increased $(\mathrm{P}<0.01)$, and similar to IL-Ra, LGWWJX significantly attenuated the expression of IL-17A from ILC3 cells with or without stimulation by IL-1 $\beta(10 \mathrm{ng} / \mathrm{mL})(\mathrm{P}<0.01)$.

\section{Discussion}

AHR is a hallmark of all forms of bronchial asthma and is even more evident in obese asthmatic individuals, which is also reflected in our present data and previous reports, as measured by increases in airway resistance in response to Mch or 5-hydroxyptrytamine in obese versus lean asthmatic animals (10-14). However, controversies are known to exist, especially in clinical settings (15-18). In addition, innate AHR can develop spontaneously in non-asthmatic obese animals and humans (19), which is also supported by our HFD-induced obesity mouse model, and no doubt contributes to the augmented AHR in obese asthma. However, little is known about the relative importance of and potential interaction between innate AHR associated with obesity and asthma-induced AHR in obese asthma, warranting further research in this field.
Up to now, only a few research groups have shed light on the mechanisms underlying obesity-induced innate AHR. However, at least two research groups have determined the important role of IL-17A, a pro-inflammatory cytokine, which is considered to take part in neutrophilic inflammation, airway remodeling, and steroid resistance in asthma, and is correlated with asthma severity (20). Shore and colleagues revealed that TNF- $\alpha$ /TNFR2 signaling was essential for the innate AHR that develops in obese mice through enhancing IL-17A expression, which was associated with complement factor D $(21,22)$. Furthermore, pulmonary rather than systemic IL-17A was essential for the development of innate AHR in obesity (21). Kim and colleagues also found that, among several potential cellular sources of IL-17A in the lungs of obese mice, ILC3 cells were much more active than others, such as Th17 and $\gamma$ T cells, and were associated with AHR (8). IL-1 $\beta$, mainly produced by macrophages, is also required for HFD-induced AHR, as the development of AHR in obese mice could be abolished with an IL-1R antagonist (8). Moreover, nutritional excess activates NACHT LRR and PYD domains-containing protein 3 (NLRP3), leading to increased IL-1 $\beta$ production (23), and activation of this obesity/NLRP3/IL-1 $\beta$ pathway could promote the expansion and activation of pulmonary IL- $17^{+} \mathrm{ILC} 3$ cells, which ultimately facilitated AHR (8). Our results in this study not only support but also extend these findings in asthmatic animals by revealing that both IL-1 $\beta$ and IL-17A in serum and BALF were elevated in obese versus lean mice 
with asthma, which paralleled the augmentation of AHR, further indicating the role of innate AHR via IL- $1 \beta$ and IL17A pathway activation.

In this study, we demonstrated for the first time that LGWWJX alleviated HFD-induced innate AHR in obese asthmatic mice. Furthermore, AHR was demonstrated in asthmatic mice and was shown to be somewhat more pronounced in obese versus non-obese mice. This discrepancy may be explained by the importance and differential expression of IL-1 $\beta$ and IL-17A between lean and obese asthmatic animals. Furthermore, it could also explain the overt differences in anti-inflammatory patterns between non-obese and obese asthma. While eosinophil infiltration in BALF was suppressed with LGWWJX treatment in non-obese mice, neutrophils and basophils were decreased in the obese mice. On the other hand, our study also noted a moderate therapeutic effect of LGWWJX through weight loss, improvement in hepatic steatosis, and possibly regulation of lipid metabolism in obese mice (6). It should be noted that only a $5 \%$ decrease in body weight is sufficient to elicit an obvious benefit in obese asthma, including improvement of lung function, from weight loss interventions in humans (1). This may be due to dilation of the reduced total lung volume and narrowed airway (24), and the fact that consumption of a HFD has been shown to inhibit pro-inflammatory cytokine responses of lymphocytes (25).

A major limitation of present data is that the ILC3 cells used in this study were of intestinal, but not pulmonary, origin. This is because the amount of ILC3 cells in mouse lung tissue is too small to be considered for in vitro experiments, while those derived from the intestines were sufficient. However, it is unclear whether there are any significant discrepancies in phenotype and function between these two cell sources, and thus, the results obtained from our in vitro experiments should be interpreted with caution.

In conclusion, our results indicated that LGWWJX exerted a protective effect on asthmatic mice, especially those with concurrent obesity, most likely through pathways including inhibition of the IL-1 $\beta / \mathrm{ILC} 3 / \mathrm{IL}-17 \mathrm{~A} / \mathrm{AHR}$ axis, as well as anti-inflammatory effects, weight loss, and the regulation of lipid metabolism. These results suggest a promising role of LGWWJX, alone or in combination with anti-inflammatory agents, for the treatment of obese asthma.

\section{Acknowledgments}

Funding: This work was supported by grants from the
National Natural Science Foundation of China (Grant No. 81503508 and 81973817 ), The capacity building of TCM evidence-based medicine research center in Longhua Hospital (19Z-2-1-b), Shanghai science and technology commission scientific research project (Grant No. 18401932200), and The 2018-2020 Three-year Action Plan for Traditional Chinese Medicine Further Development in Shanghai [Grant No. ZY(2018-2020)-CCCX-2002-06].

\section{Footnote}

Reporting Checklist: The authors have completed the ARRIVE reporting checklist. Available at http://dx.doi. org/10.21037/atm-21-1189

Data Sharing Statement: Available at http://dx.doi. org/10.21037/atm-21-1189

Conflicts of Interest: All authors have completed the ICMJE uniform disclosure form (available at http://dx.doi. org/10.21037/atm-21-1189). The authors have no conflicts of interest to declare.

Etbical Statement: The authors are accountable for all aspects of the work in ensuring that questions related to the accuracy or integrity of any part of the work are appropriately investigated and resolved. All the procedures of experiments were approved by the Animal Welfare and Ethics Group of Department of Laboratory Animal Science Fudan University (approval number: 202003003Z), in compliance with national science and technology commission of China guidelines for the care and use of animals.

Open Access Statement: This is an Open Access article distributed in accordance with the Creative Commons Attribution-NonCommercial-NoDerivs 4.0 International License (CC BY-NC-ND 4.0), which permits the noncommercial replication and distribution of the article with the strict proviso that no changes or edits are made and the original work is properly cited (including links to both the formal publication through the relevant DOI and the license). See: https://creativecommons.org/licenses/by-nc-nd/4.0/.

\section{References}

1. Miethe S, Karsonova A, Karaulov A, et al. Obesity and asthma. J Allergy Clin Immunol 2020;146:685-93. 
2. Freitas PD, Ferreira PG, Silva AG, et al. The Role of Exercise in a Weight-Loss Program on Clinical Control in Obese Adults with Asthma. A Randomized Controlled Trial. Am J Respir Crit Care Med 2017;195:32-42.

3. Scott HA, Gibson PG, Garg ML, et al. Dietary restriction and exercise improve airway inflammation and clinical outcomes in overweight and obese asthma: a randomized trial. Clin Exp Allergy 2013;43:36-49.

4. Townsend EA, Zhang Y, Xu C, et al. Active components of ginger potentiate $\beta$-agonist-induced relaxation of airway smooth muscle by modulating cytoskeletal regulatory proteins. Am J Respir Cell Mol Biol 2014;50:115-24.

5. Bak MJ, Ok S, Jun M, Jeong WS. 6-shogaol-rich extract from ginger up-regulates the antioxidant defense systems in cells and mice. Molecules 2012;17:8037-55.

6. Liu T, Yang L, Zou L, et al. Chinese medicine formula lingguizhugan decoction improves Beta-oxidation and metabolism of Fatty Acid in high-fat-diet-induced rat model of Fatty liver disease. Evid Based Complement Alternat Med 2013;2013:429738.

7. Ran S, Sun F, Song Y, et al. The Study of Dried Ginger and Linggan Wuwei Jiangxin Decoction Treatment of Cold Asthma Rats Using GC-MS Based Metabolomics. Front Pharmacol 2019;10:284.

8. Kim HY, Lee HJ, Chang YJ, et al. Interleukin-17producing innate lymphoid cells and the NLRP3 inflammasome facilitate obesity-associated airway hyperreactivity. Nat Med 2014;20:54-61.

9. Guo X, Qiu J, Tu T, et al. Induction of innate lymphoid cell-derived interleukin-22 by the transcription factor STAT3 mediates protection against intestinal infection. Immunity 2014;40:25-39.

10. Rivera-Sanchez YM, Johnston RA, Schwartzman IN, et al. Differential effects of ozone on airway and tissue mechanics in obese mice. J Appl Physiol (1985) 2004;96:2200-6.

11. Johnston RA, Zhu M, Rivera-Sanchez YM, et al. Allergic airway responses in obese mice. Am J Respir Crit Care Med 2007;176:650-8.

12. Shore SA, Rivera-Sanchez YM, Schwartzman IN, et al. Responses to ozone are increased in obese mice. J Appl Physiol (1985) 2003;95:938-45.

13. Lu FL, Johnston RA, Flynt L, et al. Increased pulmonary responses to acute ozone exposure in obese $\mathrm{db} / \mathrm{db}$ mice. Am J Physiol Lung Cell Mol Physiol 2006;290:L856-65.

14. Johnston RA, Theman TA, Lu FL, et al. Diet-induced obesity causes innate airway hyperresponsiveness to methacholine and enhances ozone-induced pulmonary inflammation. J Appl Physiol (1985) 2008;104:1727-35.

15. Kaminsky DA, Chapman DG, Holbrook JT, et al. Older age and obesity are associated with increased airway closure in response to methacholine in patients with asthma. Respirology 2019;24:638-45.

16. Kwon JW, Kim SH, Kim TB, et al. Airway hyperresponsiveness is negatively associated with obesity or overweight status in patients with asthma. Int Arch Allergy Immunol 2012;159:187-93.

17. Dixon AE, Pratley RE, Forgione PM, et al. Effects of obesity and bariatric surgery on airway hyperresponsiveness, asthma control, and inflammation. J Allergy Clin Immunol 2011;128:508-15.e152.

18. Sposato B, Scalese M, Migliorini MG, et al. Obesity can influence children's and adolescents' airway hyperresponsiveness differently. Multidiscip Respir Med 2013;8:60.

19. Shore SA, Johnston RA. Obesity and asthma. Pharmacol Ther 2006;110:83-102.

20. Ramakrishnan RK, Al Heialy S, Hamid Q. Role of IL-17 in asthma pathogenesis and its implications for the clinic. Expert Rev Respir Med 2019;13:1057-68.

21. Mathews JA, Wurmbrand AP, Ribeiro L, et al. Induction of IL-17A Precedes Development of Airway Hyperresponsiveness during Diet-Induced Obesity and Correlates with Complement Factor D. Front Immunol 2014;5:440.

22. Williams AS, Chen L, Kasahara DI, Si H, Wurmbrand AP, Shore SA. Obesity and airway responsiveness: role of TNFR2. Pulm Pharmacol Ther 2013;26:444-54.

23. Vandanmagsar B, Youm YH, Ravussin A, et al. The NLRP3 inflammasome instigates obesity-induced inflammation and insulin resistance. Nat Med 2011;17:179-88.

24. Bates JH. Physiological Mechanisms of Airway Hyperresponsiveness in Obese Asthma. Am J Respir Cell Mol Biol 2016;54:618-23.

25. de Vries A, Hazlewood L, Fitch PM, et al. High-fat feeding redirects cytokine responses and decreases allergic airway eosinophilia. Clin Exp Allergy 2009;39:731-9.

Cite this article as: Ma Z, Li C, Xue L, Zhang S, Yang Y, Zhang H, Lu Z. Linggan Wuwei Jiangxin formula ameliorates airway hyperresponsiveness through suppression of IL-1 $\beta$ and IL-17A expression in allergic asthmatic mice especially with diet-induced obesity. Ann Transl Med 2021;9(8):682. doi: 10.21037/atm-21-1189 


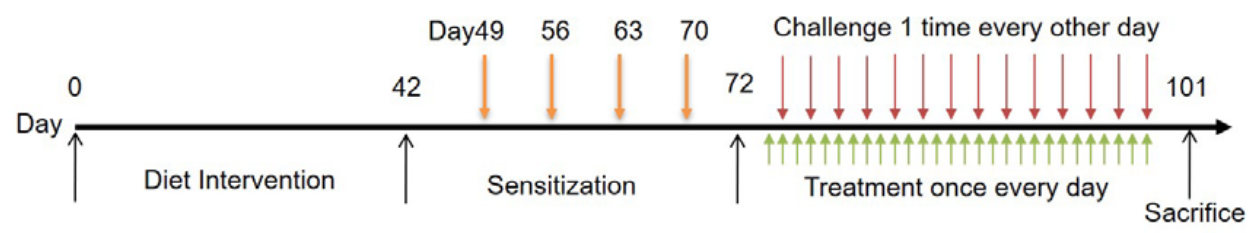

Figure S1 The experimental protocol for the establishment of the mouse model of obese asthma and intervention with Linggan Wuwei Jiangxin (LGWWJX).

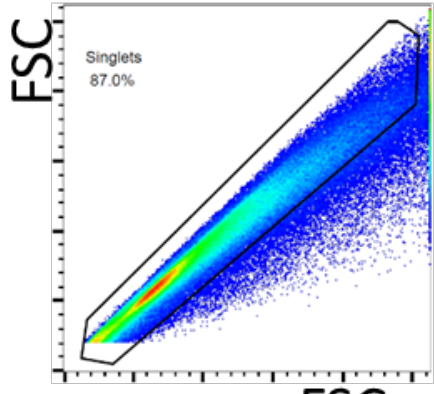

FSC

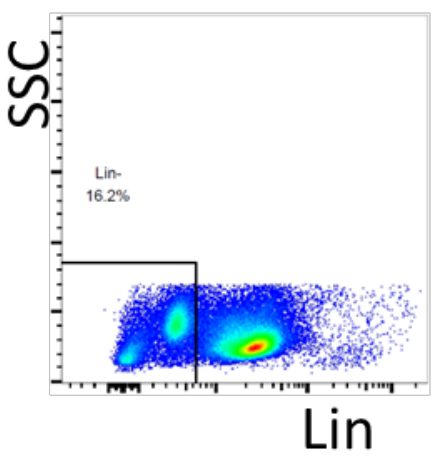

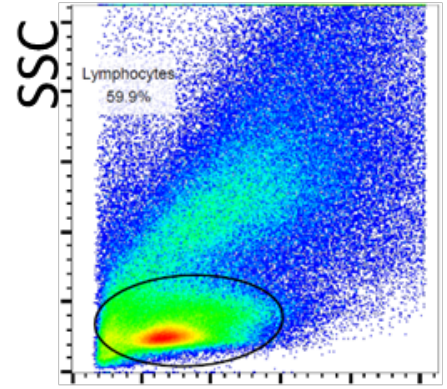

FSC

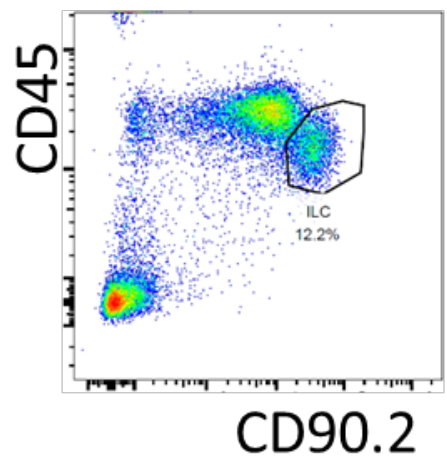

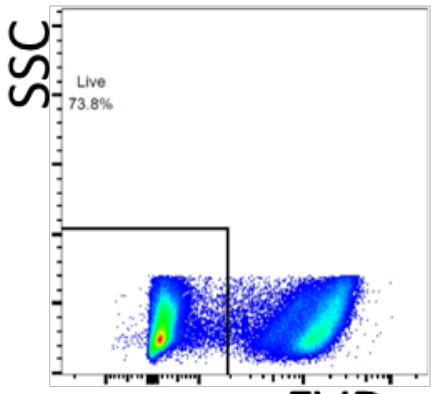

FVD

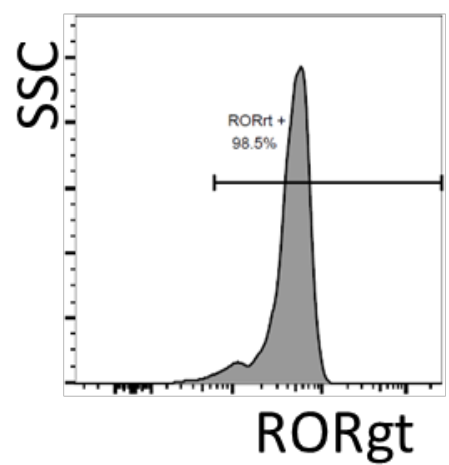

Figure S2 Innate lymphoid cell 3 (ILC3) were prepared by isolation of intestinal lamina propria lymphocytes dissected from $\mathrm{Rag}^{-/-}$mice and then sorted as Lin- CD $45^{\text {low }}$ CD $90.2^{\text {high }}$ cells and identified by nuclear ROR $\gamma$ t staining. 


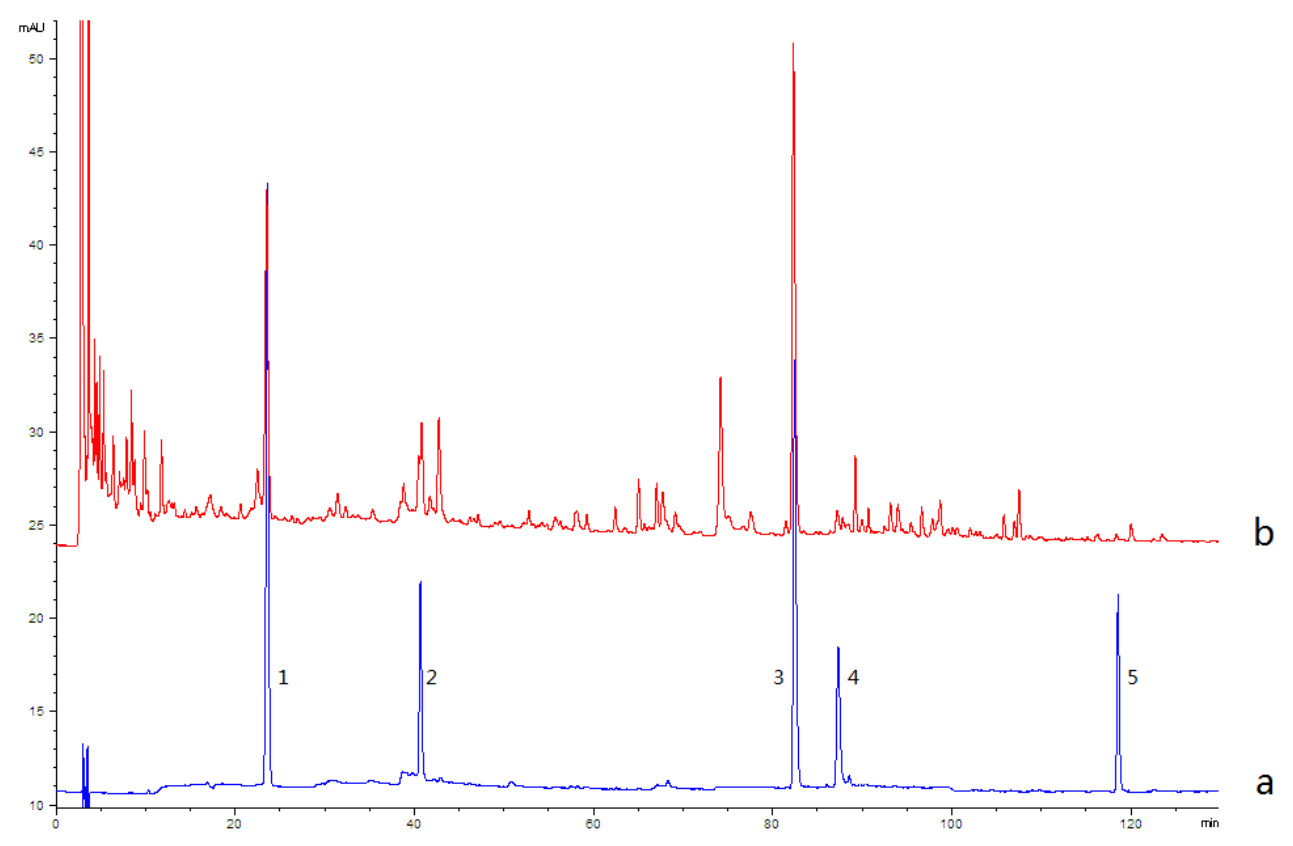

Figure S3 High performance liquid chromatography (HPLC) chromatograms of Linggan Wuwei Jiangxin (LGWWJX). Representative chromatograms of standard reference compounds (a) and LGWWJX (b) are shown. The numbers from 1 to 5 in (a) represent liquiritin, isoliquiritin, glycyrrhizinic acid, schizandrin, and deoxyschizandrin, respectively.

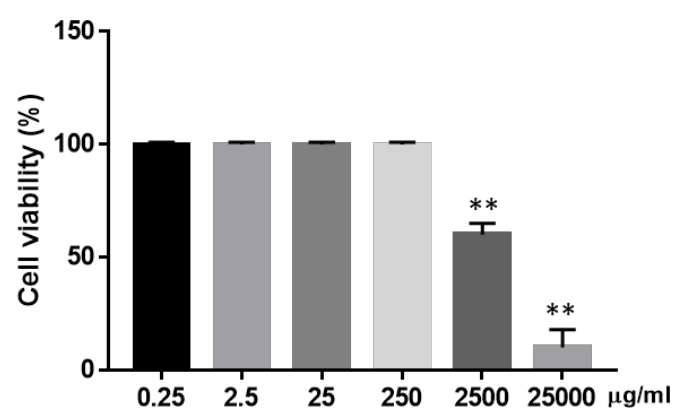

Figure S4 The concentration-dependent effects of Linggan Wuwei Jiangxin (LGWWJX) on the viability of innate lymphoid cell 3 (ILC3) cells in vitro. The values are presented as mean \pm SEM ( $n=4$ per group). ${ }^{* *} \mathrm{P}<0.01$ compared with the cell viability. 